\title{
Heat shock protein 60 (HSP60) modulates adiponectin signaling by stabilizing adiponectin receptor
}

\author{
Deling Zhang ${ }^{1,2}$, Hua Liư ${ }^{3}$, Yemin Zhang ${ }^{1,2}$, Junfeng Li ${ }^{4}$, Yalin Fu ${ }^{1,2}$, Yuyang Zheng ${ }^{1,2}$, Jie Wu ${ }^{1}$, Mingke Ma ${ }^{1,2}$, \\ Zhongyuan Wen $^{4^{*}}$ and Changhua Wang ${ }^{1,2^{*}}$
}

\begin{abstract}
Adiponectin, an adipokine produced and secreted by adipocytes, is involved in regulating the development and progression of insulin resistance, diabetes, and diabetic complications. Heat shock protein 60 (HSP60) is a molecular chaperone, most commonly presenting in mitochondria and participating in the maintenance of protein homeostasis. Accumulating studies have demonstrated that the elevated circulating HSP60 and the decreased intracellular HSP60 are closely associated with diabetic complications such as diabetic cardiomyopathy. However, the underlying mechanism remains poorly understood. In the present study, we reported that HSP60 interacted directly with adiponectin receptors. Its abundance was positively associated with adiponectin action. Furthermore, HSP60 depletion markedly mitigated the protective impacts of adiponectin on high glucose-induced oxidative stress and cell apoptosis in rat cardiac H9c2 cells. In addition, HSP60 knockdown significantly enhanced proteasome activity leading to the degradation of adiponectin receptor 1. Taken together, we showed for the first time that HSP60 interacted with adiponectin receptors and mediated adiponectin signaling through stabilizing adiponectin receptor. This in vitro study also provides an alternative explanation for mechanism by which adiponectin exerts its action.
\end{abstract}

Keywords: Heat shock protein 60, Adiponectin, Adiponectin receptor, Cardiac myocyte

\section{Background}

Adiponectin is the most abundant adipokine produced and secreted by adipocytes. Through binding with its specific receptors adiponectin receptor 1 (AdipoR1) and AdipoR2, adiponectin initiates intracellular signaling pathways and exerts promising effects in the prevention or treatment of diabetes and metabolic syndrome, cardiovascular diseases, cancers, central nervous system disorders and so on [1-4]. Previous researches have confirmed that adiponectin signaling could be mediated by adaptor protein APPLs (adaptor

\footnotetext{
* Correspondence: zhongyuan.wen@whu.edu.cn; chwang0525@whu.edu.cn ${ }^{4}$ Department of Endocrinology, Renmin Hospital of Wuhan University, Wuhan 430060, China

'Department of Pathology \& Pathophysiology, Wuhan University School of Basic Medical Sciences, Wuhan 430071, China

Full list of author information is available at the end of the article
}

protein, phosphotyrosine interacting with $\mathrm{PH}$ domain and leucine zipper) including APPL1 and APPL2 $[5,6]$. APPL1 associates with the intracellular domain of AdipoRs and positively regulates adiponectin's actions in some type of cells such as sensitizing insulin signaling in skeletal muscle cells $[5,6]$. APPL1-deficiencies in mice impair adiponectin signaling and therefore cause systemic insulin resistance [7]. In contrast, APPL2 negatively regulates adiponectin signaling by competitively interacting with AdipoRs or heterodimerizing with APPL1 [6]. The "Yin and Yang" balance between APPL1 and APPL2 orchestrates adiponectin signaling and maintains normal adiponectin function $[6,8]$.

Heat shock protein 60 (HSP60) is classically described as a molecular chaperone, most commonly presenting in mitochondria and involving in the maintenance of protein homeostasis. Under stress condition, HSP60 can translocate 
to the cytosol and cell membrane, and also secrete into blood to form serum (or circulating) HSP60 [9]. The ability of HSP60 in response to different stress greatly dependents on its localization [10]. It is noteworthy that there is an interaction between HSP60 and inflammation. HSP60 expression and secretion can be promoted in viable cells such as cardiomyocytes, adipocytes, astrocytes, and peripheral blood mononuclear cells, in response to proinflammatory cytokines as diverse as IL- $1 \beta$ and TNF- $\alpha$ [11-14]. On the other hand, serum HSP60 has been recognized as a potent inductor of proinflammatory mediators in various cells indulging innate immune cells, skeletal muscle, cardiomyocytes, and adipocytes [11, 15-17]. Furthermore, the high levels of serum HSP60 have been found in the individuals with adjuvant arthritis and atherosclerosis $[18,19]$.

Currently, accumulating evidences have linked HSP60 with diabetes mellitus and diabetic complications, although the molecular mechanisms are poorly understood [18-20]. For instance, serum HSP60 levels have been found to be significantly elevated in the patients with type 2 diabetes and morbid obesity, due to enhanced mitochondrial stress and responsible for inflammation [2022]. A modified form of highly reactive HSP60 peptide p277 (DiaPep277) has been testing to treat type 1 diabetes [23]. In addition, the elevated serum HSP60 levels also increases cardiovascular risk in obesity individuals [22]. Therefore, HSP60 may represent a potential therapeutic target for diabetes and its complications.

Interestingly, type 2 diabetic subjects exhibit the decreased expression of intracellular HSP60 in some tissues such as brain, heart, and subcutaneous adipose tissue [2426]. Importantly, the decrease in intracellular HSP60 levels is closely associated with inflammation, mitochondrial dysfunction, formation of reactive oxygen species (ROS), and insulin resistance, which are usually observed in diabetic individuals and prevented by adiponectin administration [4, 24-27]. However, the status of HSP60 in adiponectin signaling is unclear.

Here, we experimentally demonstrated that HSP60 mediated adiponectin signaling in vitro by stabilizing adiponectin receptor. This finding will undoubtedly help us to deepen our understanding of adiponectin action and explore a novel therapy strategy for diabetes and diabetic complications.

\section{Materials and methods}

\section{Antibodies and reagents}

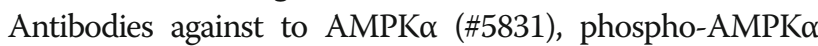
(Thr172) (\#2535), p38 MAPK (\#8690), phospho-p38 MAPK (Thr180/Tyr182) (\#9216), caspase-3 (\#9662), cleaved caspase-3 (\#9661), Myc-tag (\#2276), ubiquitin (\#3936), $\beta$ tubulin (\#2146) were from Cell Signaling Technology (Billerica, MA, USA). Antibodies against to AdipoR1 (ab70362), AdipoR2 (ab77612), and HSP60 (ab46798) were obtained from Abcam (Cambridge, MA, USA). Normal IgG (sc-2025) and secondary antibodies conjugated to horseradish peroxidase or alkaline phosphatase were purchased from Santa Cruz Biotechnology (Santa Cruz, CA, USA) or Abbiotec (San Diego, CA, USA), respectively. Recombinant mouse adiponectin (ALX-522-059) and recombinant rat adiponectin globular form (Catalog\#: SRP4593) were acquired from Enzo Life Sciences (Farmingdale, NY, USA) and SigmaAldrich (St. Louis, MO, USA), respectively. MG132 (HY13259) was obtained from MedChemExpress (Monmouth Junction, NJ, USA).

\section{Cell culture and treatment}

Rat cardiac H9c2 cell (ATCC, CRL-1446) were cultured in DMEM containing $10 \%$ fetal bovine serum (FBS) and $1 \%$ penicillin/streptomycin. Mouse liver HepIR cells (kind gifts from Drs. Feng Liu and Lily Q. Dong, UTHSCSA, USA) were cultured in MEM-alpha containing $10 \% \mathrm{FBS}$ and $0.8 \mu \mathrm{M}$ dexamethasone [6, 28]. All cells were maintained in a humidified incubator with $5 \% \mathrm{CO}_{2}$ and $95 \%$ air at $37^{\circ} \mathrm{C}$.

High glucose treatment was performed as our described previously $[29,30]$. The control group received the treatment of $5.5 \mathrm{mM}$ glucose and the identical concentration of mannitol which act as osmotic control to remove a hyperosmolar effect.

\section{Plasmid construction}

The cDNAs of full-length of mouse HSP60, mouse AdipoR1, and mouse AdipoR2 were generated by PCR and subcloned into the mammalian expression vectors pcDNA3.1 (Myctagged), or pGEX, respectively, as described previously [6].

\section{Small interfering RNAs and transfection}

The small interfering RNAs (siRNAs) targeting rat HSP60 (NM_022229.2) and mouse HSP60 (NM_010477.4) were synthesized by Genechem Co., LTD (Shanghai, China). Transfection was performed with $120 \mathrm{pM}$ of siRNA using Lipofectamine ${ }^{\circ}$ RNAiMAX Transfection Reagent (Life Technologies Corporation, Gaitherburg, MD, USA) according to the manufacturer's protocol. The most effective sequences of siRNAs and its paired control used in the experiments were as follows: rat HSP60, 5' - GAGAGG TGTGATGTTGGCTGTTGAT-3' and 5' - GAGTGTGGT AGGGTTTGTCTGAGAT - 3'; mouse HSP60, 5' -CAAA TGGAGACAAAGACATTGGGAA- $3^{\prime}$ and $5^{\prime}$-CAAAGG CAGAAACAGTTAGGATGAA-3'. Knockdown efficiency was assessed by western blot.

\section{Cell immunofluorescence}

Immunofluorescence staining was performed as our described previously $[6,30]$. Images were acquired on an Olympus IX83 laser scanning confocal microscope and analyzed by Olympus FV1200 software. 


\section{DHE staining}

The real-time formation of ROS in cells was detected by dihydroergotamine (DHE) staining as described previously [30]. Briefly, the cells were plated on the coverslips within a 24-well plate at a density of $2 \times 10^{4}$ cells/well, starved serum for $6 \mathrm{~h}$, and then treated with or without high glucose and/or other compound for the desired time. DHE (at a final concentration of $10 \mathrm{mM}$ ) was used to stain the cells at $37^{\circ} \mathrm{C}$ for $30 \mathrm{~min}$ in the dark. Cells were then rinsed once with pre-warmed PBS. DHE fluorescence was captured with fluorescence microscopy and quantified by automated image analysis.

\section{Apoptosis determination}

Terminal deoxynucleotidyl transferase-mediated dUTPbiotin nick end labeling (TUNEL) was performed to detect cells undergoing apoptosis as described by the manufacturer's protocol (Roche Applied Science, Indianapolis, IN, USA).

\section{GST pull-down, immunoprecipitation and western blot} The pull-down assay, immunoprecipitation experiments, and western blot were performed as described previously [6].

\section{Statistical analyses}

The data are presented as the means \pm SD. Differences between the groups were examined using one-way analysis of variance (ANOVA), followed by a NewmanKeuls post hoc test. The values of $p<0.05$ were considered statistically significant.

\section{Results and discussion}

\section{HSP60 associated with adiponectin receptors}

Adiponectin receptors, AdipoR1 and AdipoR2, are important members in a new family of cell surface receptor, called Progestin and AdipoQ Receptor (PAQR) family [31]. AdipoR1 is expressed ubiquitously and constitutively in most tissues and cells including adult cardiomyocytes and rat cardiac H9c2 cells [32, 33], while AdipoR2 is mainly expressed in the liver [34]. To demonstrate the association between AdipoRs with HSP60, we firstly detected the localization of AdipoRs and HSP60 in H9c2 cells and mouse liver HepIR cells [28]. Immunofluorescence staining revealed that endogenous HSP60 co-localized with endogenous AdipoR1 in H9c2 cells (Fig. 1a). Similar observation was also made when HSP60 was overexpressed in HepIR cells (Fig. 1b). To investigate whether HSP60 and AdipoRs associate directly, GST pull-down and co-immunoprecipitation assays were performed. As shown in Fig. 1c and d, endogenous AdipoR1 in H9c2 cells and AdipoR2 in HepIR cells interacted with GST-HSP60 but not with GST control proteins. Coimmunoprecipitation experiments revealed that overexpressed HSP60 interacted specifically with endogenous AdipoR1 in H9c2 cells (Fig. 1e) and endogenous AdipoR2 in
HepIR cells (Fig. 1f). These findings indicate that HSP60 interacts directly with adiponectin receptors.

\section{HSP60 mediated adiponectin action}

To understand the functional role of HSP60 in regulating adiponectin action, intracellular HSP60 protein levels were increased by overexpression (OE) or decreased by siRNA knockdown (KD), respectively. The cells were then starved serum for $6 \mathrm{~h}$, followed by stimulation with $1 \mu \mathrm{g} / \mathrm{ml}$ adiponectin for $30 \mathrm{~min}$. It has been reported that AdipoR1mediated adiponectin signaling could be activated by globular adiponectin (gADPN) whereas AdipoR2 only bind with full-length adiponectin (fADPN) [31, 34]. Therefore, H9c2 cells and HepIR cells were treated with gADPN and fADPN, respectively. We found that phosphorylation of AMPK and p38 MAPK in response to adiponectin stimulation were greatly suppressed in HSP60-KD H9c2 cells (Fig. $2 a$ and b) and HepIR cells (Fig. 2c and d) but obviously enhanced in HSP60-OE H9c2 cells (Additional file 1: Figure S1a and S1b) and HepIR cells (Additional file 1: Figure S1c and S1d), respectively. Since phosphorylation of AMPK in cardiomyocytes and p38 MAPK in hepatocytes are the markers of their activities [35, 36], our results demonstrate that HSP60 positively modulates adiponectin signaling.

In the present study, we also found that knocking down of HSP60 induced reductions of p38 MAPK and AMPK phosphorylation at basal levels (Fig. 2). Although the underlying mechanism is unclear, HSP60 has been proven to positively regulate p38 MAPK pathway in various cells [37, 38], suggesting that HSP60 plays a role in controlling p38 MAPK activity in both adiponectin-dependent and -independent mechanism. The impacts of HSP60 on AMPK activity is controversial. In cancer cells, HSP60 silencing can activate AMPK through triggering the excessive ROS production, which is beneficial for tumor progression [39, 40]. In adipose tissues, however, high-fat diet feeding induces a reduction of HSP60 protein levels and this change is not associated with any changes in AMPK activity [41]. Our finding indicates that HSP60 deficiency reduced basal AMPK phosphorylation, suggesting that adiponectin-independent mechanism is also involved in HSP60 controlled AMPK activation. Future studies are needed to dissect the specific role of HSP60 in variety of the cells residing in fat tissues in regulating AMPK activity.

\section{HSP60 knockdown mitigated the protective effects of} adiponectin on high glucose-induced oxidative stress and cell apoptosis in H9c2 cells

Hyperglycemia is a hallmark feature of both type 1 and type 2 diabetes. Previous study has evidenced that high levels of glucose induce oxidative stress and cell apoptosis in cardiomyocytes [30, 42], which can be protected by adiponectin administration [43]. Using this model, we 
A

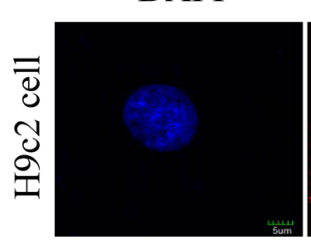

HSP60

AdipoR1
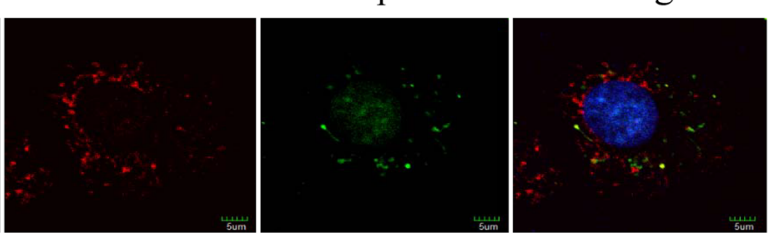

B

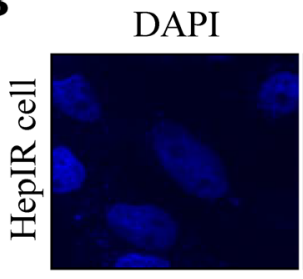

HSP60

AdipoR2

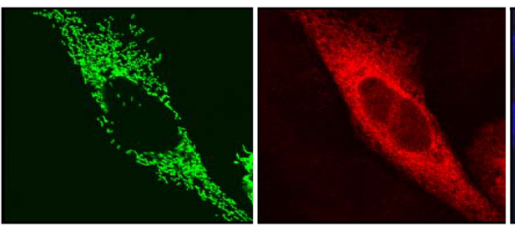

Merge

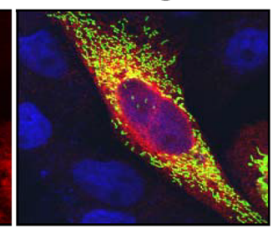

C

H9c2 cell
D
HepIR cell

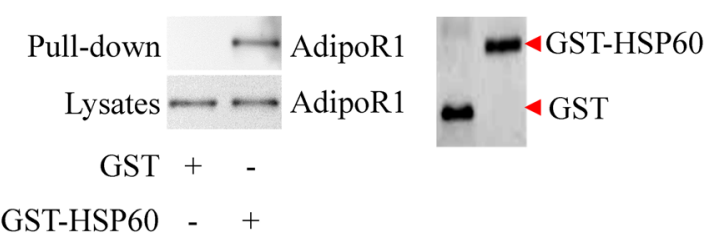

$\mathbf{E}$

H9c2 cell

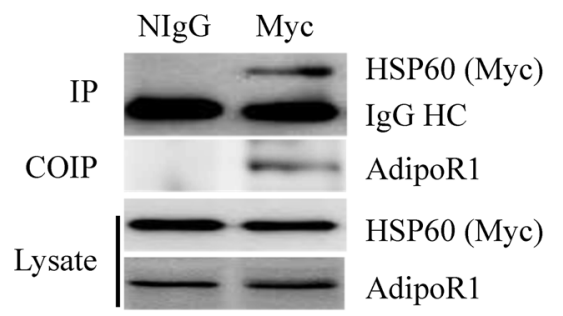

F

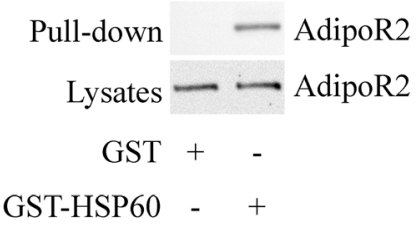

HepIR cell

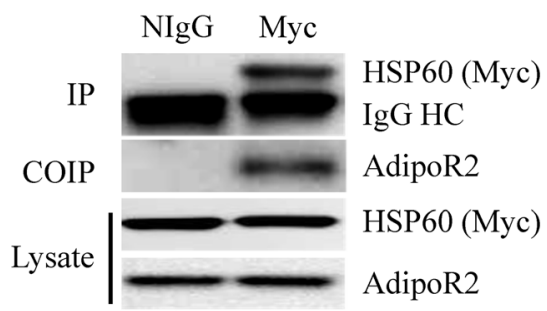

Fig. 1 HSP60 interacted with adiponectin receptors. a Colocalization of HSP60 and AdipoR1 in H9c2 cells. b Colocalization of HSP60 and AdipoR2 in HepIR cells. c Left: Pull-down of endogenous AdipoR1 with GST-HSP60 in H9c2 cells; Right: Western blot analysis of GST or GST-HSP60. d Pulldown of endogenous AdipoR2 with GST-HSP60 in HepIR cells. e Coimmunoprecipitation of AdipoR1 with HSP60 in H9c2 cells. f Coimmunoprecipitation of AdipoR2 with HSP60 in HepIR cells

wanted to further confirm the role of HSP60 in mediating adiponectin signaling.

H9c2 cells were starved serum for $6 \mathrm{~h}$, and then incubated with $5.5 \mathrm{mM}$ (normal glucose control) or $33 \mathrm{mM}$ glucose (high glucose, HG) in the presence or absence of $1 \mu \mathrm{g} / \mathrm{ml}$ of gADPN for another $48 \mathrm{~h}$. TUNEL and DHE staining assays were carried out to detect cell apoptosis and real-time formation of ROS, respectively. The cleaved caspase- 3 was detected by western blot to confirm the progression of apoptosis.

We found that HSP60 depletion significantly increased cell apoptosis, even on normal glucose (Fig. 2a, Additional file 2: Figure S2a, and Fig. 3b). This finding is consistent with previous study showing that the deletion of HSP60 in adult cardiomyocytes results in the impairment of structure and function of cardiac muscle cells [44]. Furthermore, adiponectin administration markedly inhibited HGinduced apoptosis in siRNA control cells (Fig. 3a, Additional file 2: Figure S2a, and Fig. 3b). However, these protective effects were almost completely diminished in HSP60-KD cells (Fig. 3a, Additional file 2: Figure S2a, and Fig. 3b). The similar effects on ROS formation were found in siRNA control or HSP60-KD cells treated with or without adiponectin (Fig. 3c and Additional file 2: Figure S2b). 


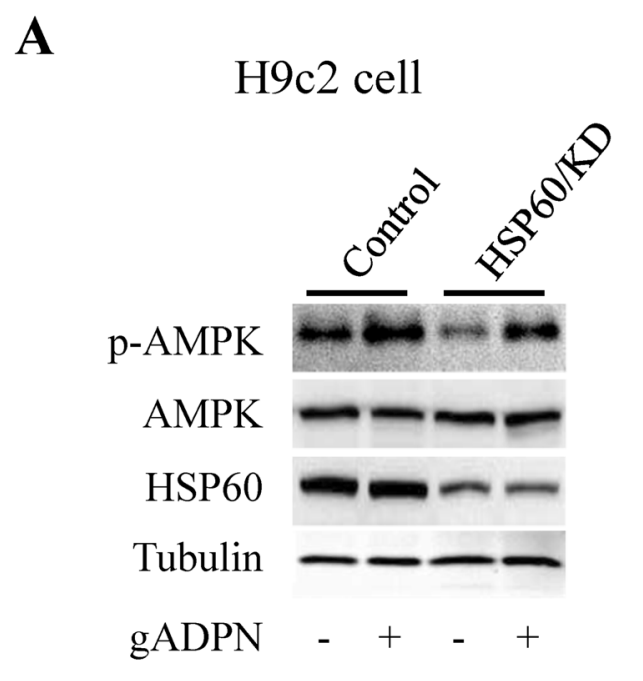

B

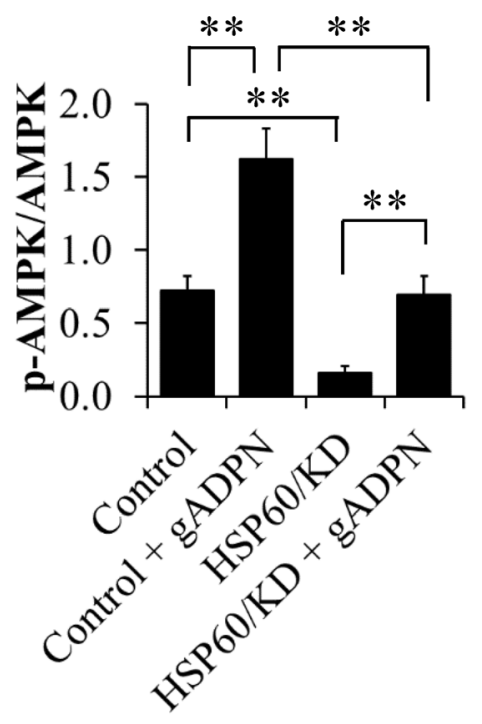

C

D
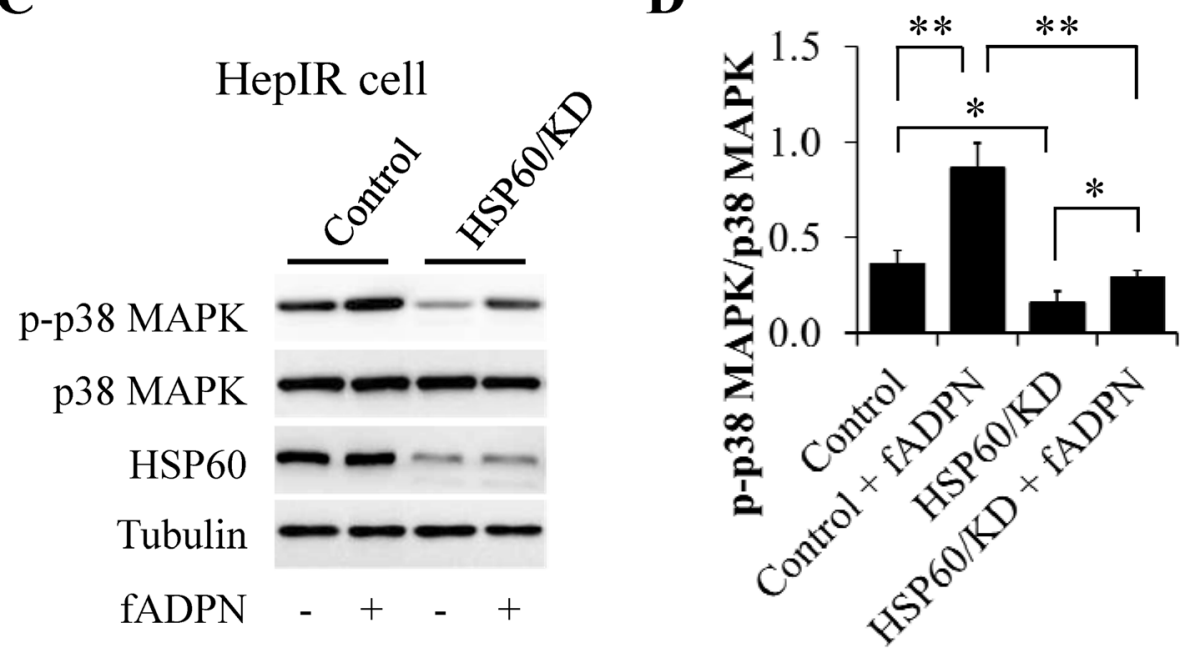

Fig. 2 HSP60 knockdown attenuated adiponectin action. a Effects of HSP60 knockdown on adiponectin-stimulated phosphorylation of AMPK in H9c2 cells. $\mathbf{b}$ Quantification of phosphor-AMPK/AMPK in (a). c Effects of HSP60 knockdown on adiponectin-stimulated phosphorylation of p38 MAPK in HepIR cells. d Quantification of phosphor-p38 MAPK/p38 MAPK in (c). Results are mean \pm SD. $n=4 .{ }^{*} P<0.05,{ }^{* *} P<0.01$ compared with the indicated group (one-way ANOVA)

These findings further confirm the HSP60 regulation on adiponectin signaling.

\section{HSP60 stabilized adiponectin receptor through a proteasome-dependent mechanism}

HSP60 has been found to positively regulate insulin-like growth factor-1 (IGF-1) signaling, through maintaining the abundance of IGF-1 receptor in cardiac muscle cells [45]. To figure out whether the similar mechanism exists in adiponectin receptors, we observed the effects of HSP60 KD on the protein levels of AdipoR1 in cardiac H9c2 cells. The cells were starved serum for $6 \mathrm{~h}$, followed by stimulation with $1 \mu \mathrm{g} / \mathrm{ml}$ of gADPN for $18 \mathrm{~h}$. We found that AdipoR1 expression was significantly reduced by HSP60 depletion but not affected by adiponectin treatment (Fig.4a and Fig. 4b), suggesting that HSP60 depletion induced AdipoR1 degradation.

It is well-known that intracellular protein degradation is mainly induced by two cellular routes: the ubiquitinproteasome system (UPS) and the autophagy-lysosome system [46]. HSP60 has been reported to modulate proteasome activity and protein ubiquitination $[45,47]$. We thus investigated the potential effects of HSP60 on the UPS. Indeed, HSP60 depletion markedly decreased the ubiquitination of total proteins (Fig. 4c). In addition, $20 \mathrm{~S}$ proteasome activity was also greatly enhanced in HSP60- 


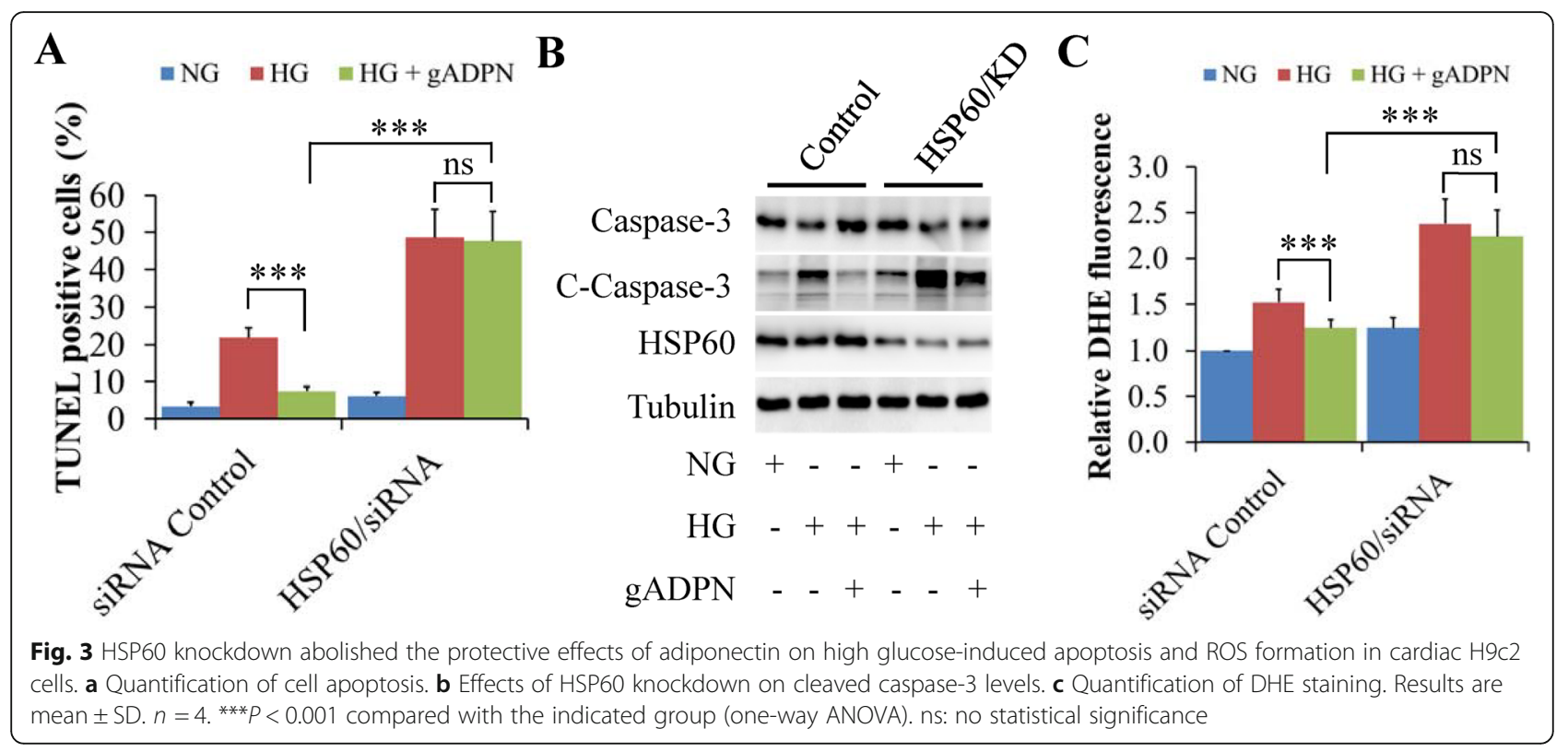

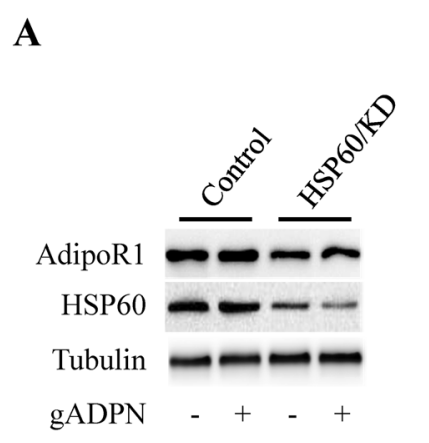

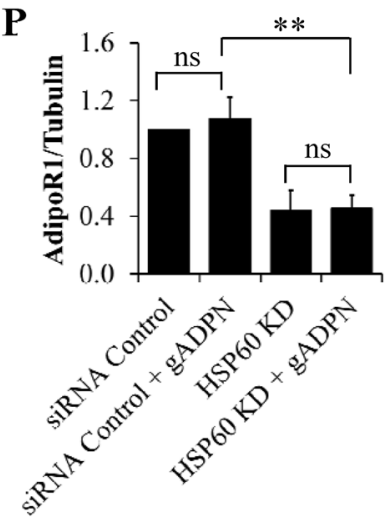

D

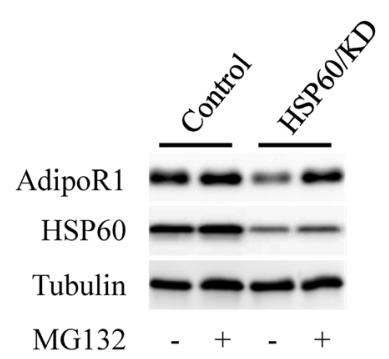

$\mathbf{E}$

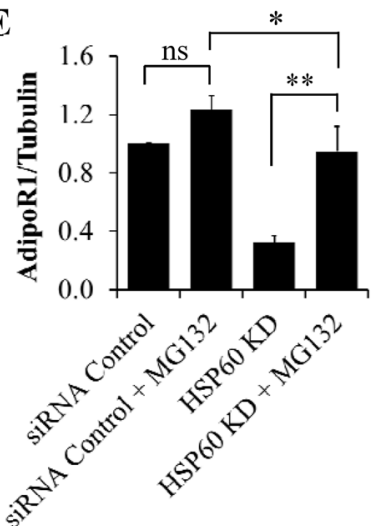

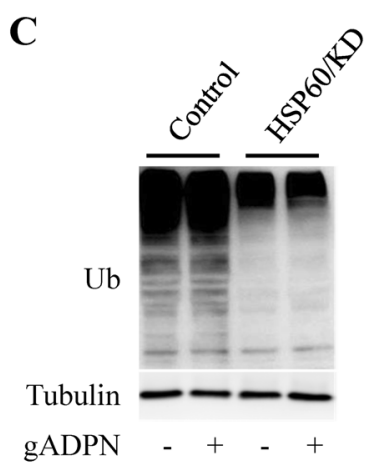

F

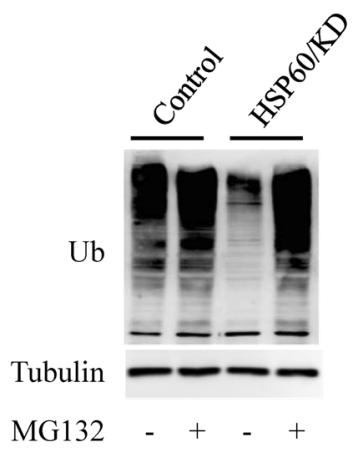

Fig. 4 HSP60 knockdown reduced AdipoR1 levels in cardiac H9c2 cells. a Effects of HSP60 knockdown on AdipoR1 expression. b Quantification of AdipoR1 protein levels in (a). c Effects of HSP60 knockdown on the ubiquitination of total proteins. $\mathbf{d}$ Effects of proteasome inhibitor MG132 on HSP60 knockdown-induced reduction of AdipoR1 expression. e Quantification of AdipoR1 protein levels in (d). F Effects of MG132 on the ubiquitination of total proteins. Results are mean \pm SD. $n=4$. ${ }^{*} P<0.05,{ }^{* *} P<0.01$ compared with the indicated group (one-way ANOVA). ns: no statistical significance 
KD H9c2 cells (Additional file 3: Figure S3). Consistent with study performed in yeast [47], these findings demonstrate that HSP60 can inhibit proteasome activity in the mammalian cells.

When H9c2 cells were starved serum for $6 \mathrm{~h}$, followed by incubation with $0.1 \mu \mathrm{M}$ of MG132, a specific proteasome inhibitor for $18 \mathrm{~h}$, we found that proteasome inhibition significantly restored HSP60 depletion-reduced protein levels of AdipoR1 (Fig. 4d and e). Proteasome inhibition also significantly increased the ubiquitination of total proteins when compared with HSP60-KD cells (Fig. 4f). These findings further suggested that HSP60 depletion-induced AdiopR1 degradation is mediated by a proteasome-dependent mechanism.

\section{Conclusion}

Adiponectin resistance, namely reduced biologic response to adiponectin in adiponectin-sensitizing tissues or cells, such as adipocytes, skeletal muscle, liver, the vasculature, and the heart [48-50], is closely associated with the development and progression of obesity, diabetes, inflammation, atherosclerosis, and cardiovascular diseases [51]. Accumulating studies have demonstrated that adiponectin resistance is related to decreased adiponectin receptor expression, reduced receptor sensitivity, and dysfunctional downstream signaling [48-50]. However, the mechanism underlying adiponectin receptor downregulation remains elusive. In the present study, we showed for the first time that HSP60 interacted with adiponectin receptors and mediated adiponectin signaling. It is highly notable that HSP60 could stabilize AdipoR1 expression through suppressing proteasome activity. This in vitro study provided an alternative explanation for the mechanism underlying adiponectin action. Given that the alteration of HSP60 protein levels have been demonstrated in diabetic complications and functionally related to hyperglycemia-induced cell injury [19], our findings will also advance our insights into basic mechanisms of HSP60 function.

In addition, our results suggest that HSP60 might represent a promising therapeutic opportunity in the diabetic complications such as diabetic cardiomyopathy. However, more in vitro and in vivo studies are necessary to further confirm our findings and to gain a full understanding of HSP60 relevance. It also needs to evaluate the impacts of HSP60 on AdipoR2 expression and figure out its clinical significance.

\section{Supplementary information}

Supplementary information accompanies this paper at https://doi.org/10. 1186/s12964-020-00546-5.

Additional file 1 Figure S1. HSP60 overexpression enhanced adiponectin action. A Effects of HSP60 overexpression on adiponectin- stimulated phosphorylation of AMPK in H9c2 cells. B Quantification of phosphor-AMPK AMPK in (A). C Effects of HSP60 overexpression on adiponectin-stimulated phosphorylation of p38 MAPK in HeplR cells. D Quantification of phosphor-p38 MAPK/p38 MAPK in (C). Results are mean \pm SD. $n=4$. ${ }^{* *} P<0.01,{ }^{* * *} P<0.001$ compared with the indicated group (one-way ANOVA).

Additional file 2: Figure S2. Effects of HSP60 knockdown on cell apoptosis and ROS formation in cardiac H9c2 cells. A Representative images showing the effects of HSP60 knockdown on apoptosis. B Representative images showing the effects of HSP60 knockdown on ROS formation.

Additional file 3: Figure S3. Effects of HSP60 knockdown on $20 \mathrm{~S}$ proteasome activity in cardiac H9c2 cells. siRNA control and HSP6O KD cells were starved serum for $6 \mathrm{~h}$. The chymotrypsin-like activity of $20 \mathrm{~S}$ proteasome was determined using synthetic fluorogenic peptide substrate Suc-LLVY-AMC as described previously [52]. Results are mean \pm SD. $n=4$. ${ }^{* * *} P<0.001$ compared with the siRNA control group (one-way ANOVA).

\section{Abbreviations}

HSP60: Heat shock protein 60; AdipoR1: Adiponectin receptor 1;

ROS: Reactive oxygen species; TUNEL: Terminal deoxynucleotidyl transferasemediated dUTP-biotin nick end labeling; UPS: Ubiquitin-proteasome system; HG: High glucose

\section{Acknowledgements}

We are very thankful to Drs. Feng Liu and Lily Q. Dong (University of Texas Health Science Center at San Antonio, UTHSCSA). The original idea for this project was from the discussion with them. Importantly, some key experiments were performed in their laboratories. Drs. Liu and Dong also kindly supply us with the key plasmids used in this study. In addition, we appreciate the helps from Wei Zhang, Libo Liu, Lisheng Fu, Yong Li, who are M.D. candidates working part time in our laboratory.

\section{Authors' contributions}

$D Z, Z W$, and CW conceived and designed the study; DZ, HL, YZ, JW, YF, YZ, and $\mathrm{JL}$ performed the experiments; $\mathrm{DZ}, \mathrm{YZ}, \mathrm{HL}$, and $\mathrm{ZW}$ analyzed and interpreted the Data; ZW and CW supervised the project and wrote the manuscript. All authors read and approved the final manuscript.

\section{Funding}

This work was supported by the National Natural Science Foundation of China (Grant No. 81170790, 81700734, 81870550) and Medical Science Advancement Program (Basic Medical Sciences) of Wuhan University (Grant No. TFJC2018001).

\section{Availability of data and materials}

All supporting data are included in this published article.

Ethics approval and consent to participate

No applicable.

Consent for publication

Not applicable.

Competing interests

The authors declare that they have no competing interests.

Author details

1Department of Pathology \& Pathophysiology, Wuhan University School of Basic Medical Sciences, Wuhan 430071, China. ${ }^{2}$ Hubei Provincial Key Laboratory of Developmentally Originated Disease, Wuhan 430071, China. ${ }^{3}$ Department of Clinical Pathology, The First People's Hospital of Lianyungang, Lianyungang 222061, China. ${ }^{4}$ Department of Endocrinology, Renmin Hospital of Wuhan University, Wuhan 430060, China. 
Received: 17 December 2019 Accepted: 6 March 2020 Published online: 09 April 2020

\section{References}

1. Bloemer J, Pinky PD, Govindarajulu M, Hong H, Judd R, Amin RH, et al. Role of Adiponectin in central nervous system disorders. Neural Plast. 2018;2018: 4593530.

2. Di Zazzo E, Polito R, Bartollino S, Nigro E, Porcile C, Bianco A, et al. Adiponectin as link factor between adipose tissue and Cancer. Int J Mol Sci. 2019;20:e839.

3. Orlando A, Nava E, Giussani M, Genovesi S. Adiponectin and cardiovascular risk. From Pathophysiology to Clinic: Focus on Children and Adolescents. Int J Mol Sci. 2019:20:e3228

4. Frankenberg ADV, Reis AF, Gerchman F. Relationships between adiponectin levels, the metabolic syndrome, and type 2 diabetes: a literature review. Arch Endocrinol Metab. 2017;61:614-22.

5. Mao X, Kikani CK, Riojas RA, Langlais P, Wang L, Ramos FJ, et al. APPL1 binds to adiponectin receptors and mediates adiponectin signalling and function. Nat Cell Biol. 2006;8:516-23.

6. Wang $C$, Xin X, Xiang R, Ramos FJ, Liu M, Lee HJ, et al. Yin-Yang regulation of adiponectin signaling by APPL isoforms in muscle cells. J Biol Chem. 2009:284:31608-15.

7. Ryu J, Galan AK, Xin X, Dong F, Abdul-Ghani MA, Zhou L, et al. APPL1 potentiates insulin sensitivity by facilitating the binding of IRS1/2 to the insulin receptor. Cell Rep. 2014;7:1227-38.

8. Ruan H, Dong LQ. Adiponectin signaling and function in insulin target tissues. J Mol Cell Biol. 2016;8:101-9.

9. Grundtman C, Kreutmayer SB, Almanzar G, Wick MC, Wick G. Heat shock protein 60 and immune inflammatory responses in atherosclerosis. Arterioscler Thromb Vasc Biol. 2011;31:960-8.

10. Krause M, Heck TG, Bittencourt A, Scomazzon SP, Newsholme P, Curi R, et al. The chaperone balance hypothesis: the importance of the extracellular to intracellular HSP70 ratio to inflammation-driven type 2 diabetes, the effect of exercise, and the implications for clinical management. Mediat Inflamm. 2015:2015:249205.

11. Marker T, Sell H, Zillessen P, Glode A, Kriebel J, Ouwens DM, et al. Heat shock protein 60 as a mediator of adipose tissue inflammation and insulin resistance. Diabetes. 2012;61:615-25.

12. Bajramovic JJ, Bsibsi M, Geutskens SB, Hassankhan R, Verhulst KC, Stege GJ, et al. Differential expression of stress proteins in human adult astrocytes in response to cytokines. J Neuroimmunol. 2000;106:14-22.

13. Gupta S, Knowlton AA. HSP60 trafficking in adult cardiac myocytes: role of the exosomal pathway. Am J Physiol Heart Circ Physiol. 2007;292:H3052-6.

14. Ireland HE, Leoni F, Altaie O, Birch CS, Coleman RC, Hunter-Lavin C, et al. Measuring the secretion of heat shock proteins from cells. Methods. 2007; 43:176-83.

15. Habich C, Baumgart $\mathrm{K}$, Kolb H, Burkart V. The receptor for heat shock protein 60 on macrophages is saturable, specific, and distinct from receptors for other heat shock proteins. J Immunol. 2002;168:569-76.

16. Gulden E, Mollerus S, Bruggemann J, Burkart V, Habich C. Heat shock protein 60 induces inflammatory mediators in mouse adipocytes. FEBS Lett. 2008;582:2731-6.

17. Tian J, Guo X, Liu XM, Liu L, Weng QF, Dong SJ, et al. Extracellular HSP60 induces inflammation through activating and up-regulating TLRs in cardiomyocytes. Cardiovasc Res. 2013;98:391-401.

18. Juwono J, Martinus RD. Does Hsp60 provide a link between mitochondrial stress and inflammation in diabetes mellitus? J Diabetes Res. 2016;2016: 8017571.

19. Bellini S, Barutta F, Mastrocola R, Imperatore L, Bruno G, Gruden G. Heat shock proteins in vascular diabetic complications: review and future perspective. Int J Mol Sci. 2017;18:e2709.

20. Imatoh T, Sugie T, Miyazaki M, Tanihara S, Baba M, Momose Y, et al. Is heat shock protein 60 associated with type 2 diabetes mellitus? Diabetes Res Clin Pract. 2009;85:208-12.

21. Yuan J, Dunn P, Martinus RD. Detection of Hsp60 in saliva and serum from type 2 diabetic and non-diabetic control subjects. Cell Stress Chaperones. 2011;16:689-93.

22. Sell H, Poitou C, Habich C, Bouillot JL, Eckel J, Clement K. Heat shock protein 60 in obesity: effect of bariatric surgery and its relation to inflammation and cardiovascular risk. Obesity (Silver Spring). 2017;25:2108-14.
23. Raz I, Ziegler AG, Linn T, Schernthaner G, Bonnici F, Distiller LA, et al. Treatment of recent-onset type 1 diabetic patients with DiaPep277: results of a double-blind, placebo-controlled, randomized phase 3 trial. Diabetes Care. 2014;37:1392-400

24. Kleinridders A, Lauritzen HP, Ussar S, Christensen JH, Mori MA, Bross P, et al. Leptin regulation of $\mathrm{Hsp60}$ impacts hypothalamic insulin signaling. J Clin Invest. 2013;123:4667-80.

25. Oksala NK, Laaksonen DE, Lappalainen J, Khanna S, Nakao C, Hanninen O, et al. Heat shock protein 60 response to exercise in diabetes: effects of alpha-lipoic acid supplementation. J Diabetes Complicat. 2006;20:257-61.

26. Khadir A, Kavalakatt S, Cherian P, Warsame S, Abubaker JA, Dehbi M, et al. Physical Exercise Enhanced Heat Shock Protein 60 Expression and Attenuated Inflammation in the Adipose Tissue of Human Diabetic Obese Front Endocrinol (Lausanne). 2018;9:16.

27. Achari AE, Jain SK. Adiponectin, a therapeutic target for obesity, diabetes, and endothelial dysfunction. Int J Mol Sci. 2017;18:e1321.

28. Liu M, Zhou L, Wei L, Villarreal R, Yang X, Hu D, et al. Phosphorylation of adaptor protein containing pleckstrin homology domain, phosphotyrosine binding domain, and leucine zipper motif 1 (APPL1) at Ser430 mediates endoplasmic reticulum (ER) stress-induced insulin resistance in hepatocytes. Jiol Chem. 2012;287:26087-93.

29. Sun S, Liu L, Tian X, Guo Y, Cao Y, Mei Y, et al. Icariin attenuates high glucose-induced apoptosis, oxidative stress, and inflammation in human umbilical venous endothelial cells. Planta Med. 2019;85:473-82.

30. Jiang P, Zhang D, Qiu H, Yi X, Zhang Y, Cao Y, et al. Tiron ameliorates high glucose-induced cardiac myocyte apoptosis by PKCdelta-dependent inhibition of osteopontin. Clin Exp Pharmacol Physiol. 2017;44:760-70.

31. Yamauchi T, Iwabu M, Okada-Iwabu M, Kadowaki T. Adiponectin receptors: a review of their structure, function and how they work. Best Pract Res Clin Endocrinol Metab. 2014:28:15-23.

32. Ding G, Qin Q, He N, Francis-David SC, Hou J, Liu J, et al. Adiponectin and its receptors are expressed in adult ventricular cardiomyocytes and upregulated by activation of peroxisome proliferator-activated receptor gamma. J Mol Cell Cardiol. 2007:43:73-84.

33. Arnold N, Mahmood A, Ramdas M, Ehlinger PP, Pulakat L. Regulation of the cardioprotective adiponectin and its receptor AdipoR1 by salt. Can J Physiol Pharmacol. 2017;95:305-9.

34. Kadowaki T, Yamauchi T, Kubota N, Hara K, Ueki K, Tobe K. Adiponectin and adiponectin receptors in insulin resistance, diabetes, and the metabolic syndrome. J Clin Invest. 2006;116:1784-92.

35. Park M, Sabetski A, Kwan Chan Y, Turdi S, Sweeney G. Palmitate induces ER stress and autophagy in $\mathrm{H} 9 \mathrm{c} 2$ cells: implications for apoptosis and adiponectin resistance. J Cell Physiol. 2015;230:630-9.

36. Wanninger J, Neumeier M, Weigert J, Bauer S, Weiss TS, Schaffler A, et al. Adiponectin-stimulated CXCL8 release in primary human hepatocytes is regulated by ERK1/ERK2, p38 MAPK, NF-kappaB, and STAT3 signaling pathways. Am J Physiol Gastrointest Liver Physiol. 2009:297:G611-8.

37. Swaroop S, Sengupta N, Suryawanshi AR, Adlakha YK, Basu A. HSP60 plays a regulatory role in IL-1beta-induced microglial inflammation via TLR4-p38 MAPK axis. J Neuroinflammation. 2016;13:27.

38. Liu B, Li S, Xiu B, Zhang Y, Zhou Y, Yang Q, et al. C-terminus of heat shock protein 60 can activate macrophages by lectin-like oxidized low-density lipoprotein receptor 1. Biochem Biophys Res Commun. 2019:508:1113-9.

39. Tang H, Chen Y, Liu X, Wang S, LV Y, Wu D, et al. Downregulation of HSP60 disrupts mitochondrial proteostasis to promote tumorigenesis and progression in clear cell renal cell carcinoma. Oncotarget. 2016;7:38822-34.

40. Guo J, Li X, Zhang W, Chen Y, Zhu S, Chen L, et al. HSP60-regulated mitochondrial Proteostasis and protein translation promote tumor growth of ovarian Cancer. Sci Rep. 2019:9:12628.

41. Sutherland LN, Capozzi LC, Turchinsky NJ, Bell RC, Wright DC. Time course of high-fat diet-induced reductions in adipose tissue mitochondrial proteins: potential mechanisms and the relationship to glucose intolerance. Am J Physiol Endocrinol Metab. 2008;295:E1076-83.

42. Davargaon RS, Sambe AD, Muthangi WS. Trolox prevents high glucoseinduced apoptosis in rat myocardial H9c2 cells by regulating GLUT-4 and antioxidant defense mechanism. IUBMB Life. 2019;71:1876-95.

43. Li H, Yao W, Irwin MG, Wang T, Wang S, Zhang L, et al. Adiponectin ameliorates hyperglycemia-induced cardiac hypertrophy and dysfunction by concomitantly activating Nrf2 and Brg1. Free Radic Biol Med. 2015;84:311-21.

44. Fan F, Duan $Y$, Yang F, Trexler C, Wang H, Huang $L$, et al. Deletion of heat shock protein 60 in adult mouse cardiomyocytes perturbs mitochondrial 
protein homeostasis and causes heart failure. Cell Death Differ. 2020;27:587600.

45. Shan YX, Yang TL, Mestril R, Wang PH. Hsp10 and Hsp60 suppress ubiquitination of insulin-like growth factor-1 receptor and augment insulinlike growth factor-1 receptor signaling in cardiac muscle: implications on decreased myocardial protection in diabetic cardiomyopathy. J Biol Chem. 2003;278:45492-8.

46. Wang $C$, Wang $X$. The interplay between autophagy and the ubiquitinproteasome system in cardiac proteotoxicity. Biochim Biophys Acta. 1852; 2015:188-94.

47. Kalderon B, Kogan G, Bubis E, Pines O. Cytosolic Hsp60 can modulate proteasome activity in yeast. J Biol Chem. 2015;290:3542-51.

48. Wang Y, Ma XL, Lau WB. Cardiovascular Adiponectin resistance: the critical role of Adiponectin receptor modification. Trends Endocrinol Metab. 2017; 28:519-30.

49. Sente T, Van Berendoncks AM, Hoymans VY, Vrints CJ. Adiponectin resistance in skeletal muscle: pathophysiological implications in chronic heart failure. J Cachexia Sarcopenia Muscle. 2016;7:261-74.

50. Engin A. Adiponectin-resistance in obesity. Adv Exp Med Biol. 2017;960:415-41.

51. Fang H, Judd RL. Adiponectin regulation and function. Compr Physiol. 2018; 8:1031-63.

52. Ye M, Qiu H, Cao Y, Zhang M, Mi Y, Yu J, et al. Curcumin improves Palmitate-induced insulin resistance in human umbilical vein endothelial cells by maintaining Proteostasis in endoplasmic reticulum. Front Pharmacol. 2017;8:148.

\section{Publisher's Note}

Springer Nature remains neutral with regard to jurisdictional claims in published maps and institutional affiliations.

Ready to submit your research? Choose BMC and benefit from:

- fast, convenient online submission

- thorough peer review by experienced researchers in your field

- rapid publication on acceptance

- support for research data, including large and complex data types

- gold Open Access which fosters wider collaboration and increased citations

- maximum visibility for your research: over $100 \mathrm{M}$ website views per year

At $\mathrm{BMC}$, research is always in progress.

Learn more biomedcentral.com/submissions 